\title{
COINFECCIÓN POR Mycobacterium fortuitum Y Mycobacterium tuberculosis EN ABSCESOS ESPLÉNICOS EN UN PACIENTE CON VIH
}

\author{
Leslie Soto-Arquíñigo ${ }^{1,2, a}$, Manuel García-Pareja1,3,b, Eduardo Gotuzzo-Herencia ${ }^{1,2, a}$, \\ Pedro Legua-Leiva ${ }^{1,2, a}$, Manuel Sánchez-Herrera ${ }^{4, b}$
}

\begin{abstract}
RESUMEN
Los pacientes con VIH son susceptibles a la infección por micobacterias. En el caso de las micobacterias de crecimiento rápido, grupo al que pertenece el Mycobacterium fortuitum ( $M$. fortuitum), se han descrito infecciones en la piel, pulmones, ganglios linfáticos y enfermedad diseminada. Presentamos el caso de un paciente varón de 43 años, con diagnóstico previo de $\mathrm{VIH}$ en tratamiento antirretroviral, que acude por fiebre, astenia, pérdida de peso y diarrea crónica. Se realiza tomografía abdominal y se evidencia hepatoesplenomegalia con lesiones nodulares en el bazo. El cultivo esplénico, finalmente, fue positivo para M. fortuitum, con PCR positivo a Mycobacterium tuberculosis. Los protocolos actuales de tratamiento para este tipo de infecciones se basan en la susceptibilidad mostrada en los cultivos realizados. En lo que respecta a coinfecciones entre M. fortuitum y Mycobacterium tuberculosis, en pacientes VIH positivos, la información es aun menor.
\end{abstract}

Palabras clave: Mycobacterium fortuitum, Mycobacterium tuberculosis; VIH; absceso (Fuente: DeCS BIREME).

CO-INFECTION BY Mycobacterium fortuitum AND Mycobacterium tuberculosis IN
SPLENIC ABSCESSES IN A PATIENT WITH HIV

\begin{abstract}
Patients with HIV are susceptible to mycobacterium infection. In the case of fast-growing mycobacteria, the group to which Mycobacterium fortuitum (M. fortuitum) belongs, infections have been described in the skin, lungs, lymph nodes and disseminated disease. We present the case of a 43-year-old male patient with pre-diagnosis of HIV in antiretroviral therapy, which comes as a fever, asthenia, weight loss and chronic diarrhea. Abdominal tomography is performed and hepatosplenomegaly is evidenced with nodular lesions in the spleen. The splenic culture was finally positive for $M$. Fortuitum, with positive PCR to Mycobacterium tuberculosis. The current treatment protocols for this type of infection are based on the susceptibility shown in the cultures performed. With regard to coinfections between M. Fortuitum and Mycobacterium tuberculosis, in HIV positive patients, there are even less information.
\end{abstract}

Key words: Mycobacterium fortuitum, Mycobacterium tuberculosis; HIV; abscess (Source: MeSH NLM).

\section{INTRODUCCIÓN}

El Mycobacterium fortuitum ( $M$. fortuitum) es una micobacteria ubicua. Taxonómicamente, forma parte de las micobacterias no tuberculosas de crecimiento rápido (siete días en medios sólidos), no pigmentadas ${ }^{(1)}$. Se ha descrito como agente causal de infecciones de piel y tejidos blandos, además de óseas y articulares como las más frecuentes $y$, en menor frecuencia, pulmonares e infecciones diseminadas ${ }^{(2)}$ tanto sintomáticas como asintomáticas. Para su diagnóstico es requerido un alto índice de sospecha conociendo los factores predisponentes y criterios diagnósticos de acuerdo a lo postulado por la Sociedad Americana de Tórax (ATS por sus siglas en inglés) ${ }^{(3)}$. La dificultad al momento de realizar el diagnóstico radica en elegir el esquema de tratamiento ya que no hay un único esquema aceptado, además, es conocido su alta resistencia a los antituberculosos usuales y muchos otros antibióticos ${ }^{(1,2)}$.

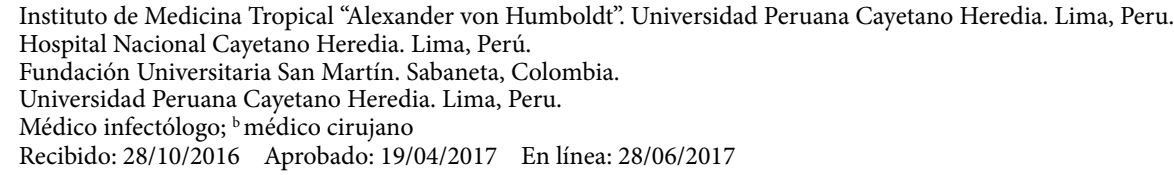

Citar como: Citar como: Soto-Arquíñigo L, García-Pareja M, Gotuzzo-Herencia E, Legua-Leiva P, Sánchez-Herrera M. Confección por Mycobacterium fortuitum y Mycobacterium tuberculosis en abscesos esplénicos en un paciente con VIH. Rev Peru Med Exp Salud Publica. 2017;34(2): 328-31. doi: 10.17843/rpmesp.2017.342.2470 
A continuación, presentamos el caso de un paciente seropositivo para $\mathrm{VIH}$, con infección diseminada por Mycobacterium fortuitum y Mycobacterium tuberculosis.

\section{REPORTE DE CASO}

Paciente varón de 43 años natural de Lima procedente de Caracas, Venezuela; con diagnóstico previo de VIH, con diarrea crónica, conteo CD4 de 108 células/mL y carga viral mayor de 500000 copias $/ \mathrm{mL}$. Se había iniciado terapia HAART con efavirenz, lamivudina y zidovudina; durante el tratamiento presenta fiebre de $39^{\circ} \mathrm{C}$, astenia, pérdida de peso y persistencia de las deposiciones diarreicas. Hospitalizado en Venezuela, inicialmente, presentaba pancitopenia, PPD $0 \mathrm{~mm}$, radiografía de tórax sin hallazgos relevantes, y una tomografía abdominal con hepatoesplenomegalia con lesiones nodulares en el bazo (Figura 1). El estudio anatomopatológico de las muestras de hígado y bazo evidenció hepatitis granulomatosa e inflamación crónica granulomatosa esplénica. Adicionalmente, la biopsia de ganglio inguinal fue inespecífica (inflamación crónica granulomatosa). Las coloraciones para hongos y BAAR de dichas muestras fueron negativas. Debido a esto, se decide iniciar tratamiento antituberculoso y anfotericina B E.V. sin presentar mejoría clínica.

Estudios imagenológicos posteriores de tórax (radiografía y tomografía) evidenciaron infiltrado miliar sugerente de TB miliar. Se adiciona terapia para NIH, PCP, MAC, TVP. Como, a pesar de esto, no hay mejoría, se sospecha de tuberculosis multidrogorresistente (TB MDR). Se realiza una nueva tomografía abdominal que muestra imágenes hipodensas en hígado y bazo; asimismo, una tomografía

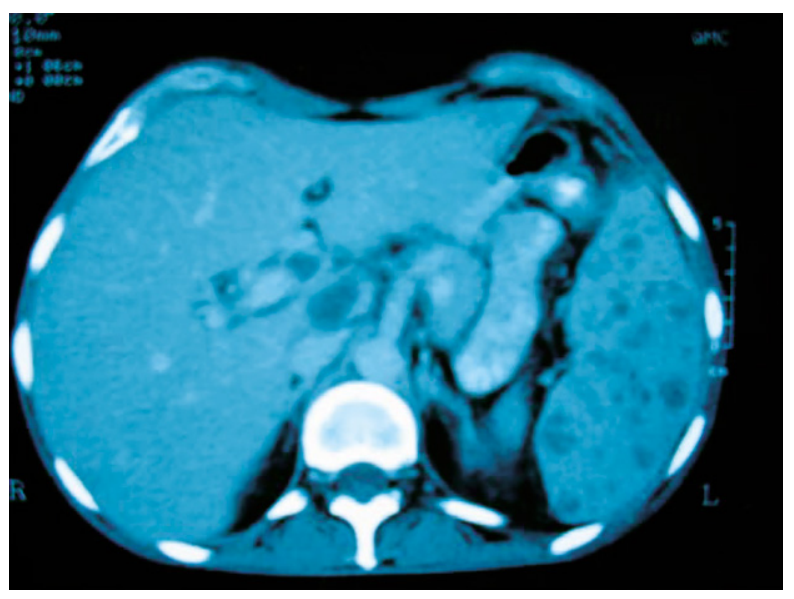

Figura 1. Tomografía abdominal donde se evidencia hepatoesplenomegalia y lesiones nodulares en bazo pélvica evidencia un absceso isquiopúbico izquierdo. La muestra de este absceso fue sometida a PCR que resulto positivo para Mycobacterium tuberculosis. Se prolonga la primera fase de antituberculosos y se añade amikacina y ofloxacina ante la posibilidad de TB MDR. EI paciente, posterior a esto, viaja a Lima y se hospitaliza en el Hospital Nacional Cayetano Heredia.

En su evaluación inicial se palpa hepatoesplenomegalia sin linfadenopatías, herida quirúrgica (biopsia previa) en región inguinal derecha con secreción serosa. Se realiza nueva tomografía abdominal con bazo aumentado de tamaño, y múltiples lesiones nodulares e hipodensas distribuidas en todo el parénquima esplénico. Se realizó un estudio de sensibilidad de TB que demostró ser sensible a rifampicina y resistente a isoniazida, pirazinamida y etambutol.

Se procede a esplenectomía total (Figura 2) y estudios anatomopatológicos arrojaron BAAR positivo. Se inicia tratamiento con levofloxacina, rifampicina, etionamida, capreomicina y amoxicilina/ ácido clavulánico. El resultado de cultivo esplénico fue positivo para Mycobacterium fortuitum, con PCR positivo a Mycobacterium tuberculosis. Por tales motivos, se instaura el esquema de manejo con linezolid, moxifloxacino, rifampicina, capreomicina, doxiciclina y amoxicilina/ácido clavulánico; esquema con el cual el paciente presentó evolución favorable. Se culmina el tratamiento con pirazinamida, rifampicina, capreomicina, cicloserina y ciprofloxacina, amoxicilina/ ácido clavulánico. Posteriormente, se da de alta médica al paciente quien sale con un conteo de CD4 de 455 células $/ \mathrm{mL}$.

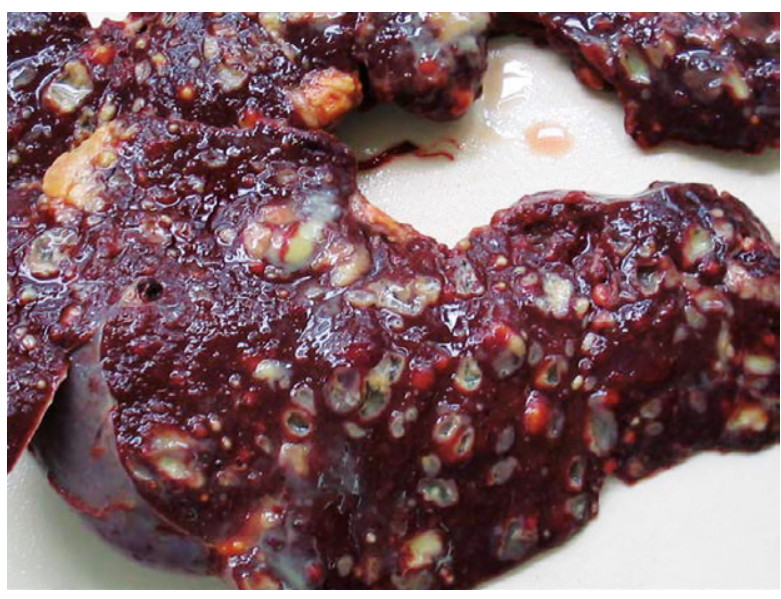

Figura 2. Muestra anatomopatológica de esplenectomía total donde se evidencian múltiples lesiones nodulares 


\section{DISCUSIÓN}

Las micobacterias no tuberculosas son organismos ambientales ubicuos que rara vez son causa de enfermedad en humanos (4). En países en vías de desarrollo, se presume que la mayoría de síntomas pulmonares que aparenten ser causados por micobacterias, son causados por M. tuberculosis. Esto se debe a la falta de herramientas diagnósticas en áreas de limitado recursos y la naturaleza endémica del patógeno ${ }^{(7,8)}$. Esto causa que muchas veces se realice un mal diagnóstico de la enfermedad, asumiéndose que se trata de $M$. tuberculosis cuando en realidad se trata de otro tipo de micobacteria. Con el advenimiento del VIH y terapias inmunosupresoras la prevalencia de micobacterias no tuberculosas ha aumentado considerablemente.

EI M. fortuitum, inicialmente aislado de una tortuga en 1905 , es una micobacteria no tuberculosa atípica, patógeno de bajo grado del cual según algunos estudios su prevalencia en pacientes $\mathrm{VIH}$ es alrededor del $21 \%$, con rangos tan amplios que van del 7 al 49\% según la muestra analizada de las infecciones por micobacterias no tuberculosas ${ }^{(5,6)}$. M. fortuitum ha sido implicado en infecciones cutáneas, protésicas y, en raras ocasiones, pulmonares ${ }^{(9-11)}$, excepto en personas con vómito crónico, enfermedad esofágica o inmunosupresión. En un paciente sin estas comorbilidades, es generalmente considerado como un colonizador ${ }^{(10)}$. Se ha visto, además, asociado a infecciones luego de procedimientos quirúrgicos como liposucción, colocación de siliconas y marcapasos.

Un nivel de linfocitos T CD4+ menor a 100 celulas $/ \mathrm{mcL}$ ha sido identificado como factor de riesgo significativo para el desarrollo de enfermedades por micobacterias no tuberculosas. Como este tipo de linfocitos se encargan de la mayor producción de interferon-gamma y de otras citoquinas, se cree que este interferón es de vital importancia para el control de estas infecciones ${ }^{(4)}$.

Para su diagnóstico se hace necesario tener un alto índice de sospecha clínica, conociendo los factores predisponentes del paciente para este tipo de infecciones. Según lo propuesto por la Sociedad Americana de Tórax se consideran como factores predisponentes: CD4 $<50$ células $/ \mathrm{mL}$, antecedente de tuberculosis y neumopatías (bronquiectasias) ${ }^{(3)}$.

En la literatura se han reportado aislamientos a nivel pulmonar, piel y tejidos blandos y hueso, causando infecciones tanto en el ámbito nosocomial y comunitario $^{(2,3,5,6)}$. Debido a los pocos casos reportados, se ha hecho difícil encontrar una guía universal de tratamiento. Se han analizado varios esquemas con buena respuesta terapéutica, todos basados en pruebas de sensibilidad. Se ha demostrado que el $M$. fortuitum tiene susceptibilidad a amikacina (100\%), ciprofloxacina y ofloxacina (100\%), sulfonamidas (100\%), cefoxitina (50\%), imipenem (100\%), claritromicina $(80 \%)$ y doxiciclina $(50 \%){ }^{(3,6)}$. A pesar de que los estudios de la actividad de macrolidos contra el $M$. fortuitum, encuentran que el $80 \%$ de los asilamientos son susceptibles a claritromicina, existe la preocupación del desarrollo de resistencia a estos medicamentos debido a la reciente detección del gen erm (39 y 41) en estas especies. Este gen codifica una enzima metilasa que adhiere 1 o 2 grupos metil al 23s rRNA, previniendo que los macrolidos se unan al ribosoma bacteriano ${ }^{(12,13)}$. El tiempo de tratamiento antibiótico oscila entre los 4-6 meses. En los casos en donde el patógeno sea resistente o el paciente no pueda tolerar los antibióticos, se recomienda la escisión quirúrgica ${ }^{(14)}$.

Nuestro paciente, por su presentación inusual, fue un gran reto diagnóstico, además de terapéutico, dada la confección con TB MDR. No se encontró reporte en las bases de datos analizadas de infecciones en bazo por $M$. fortuitum. Se hace necesario, entonces, comunicar este tipo de casos para, en un futuro, contar con la información necesaria y suficiente para la realización de un consenso diagnóstico y terapéutico que facilite el manejo correcto para este tipo de pacientes. Asimismo, pensar en coinfecciones con micobacterias atípicas en pacientes con tuberculosis resistente a la medicación.

Fuentes de financiamiento: autofinanciado.

Conflictos de interés: no existen conflictos de interés.

Contribución de los autores: LSA participo en la redacción, revisión crítica del artículo y aprobación de su versión final MGP, MSH participaron en la redaccion del articulo; EGH y PLL participaron en la revisión crítica del artículo.

\section{REFERENCIAS BIBLIOGRÁFICAS}

1. Brown-Elliott BA, Wallace RJ. Clinical and Taxonomic Status of Pathogenic Nonpigmented or Late-Pigmenting Rapidly Growing Mycobacteria. Clinical Microbiology Reviews. 2002;15(4):716-746. doi: $10.1128 /$ CMR.15.4.716746.2002
2. Brown-Elliott BA, Wallace $\mathrm{Jr}$ RJ. Infections caused by nontuberculous mycobacteria. In: Mandell, Bennett, Dolin, eds. Mandell, Douglas and Bennett's Principles and Practice of Infectious Diseases. 7th ed. Vol. 2. Elsevier Churchill Livingstone Inc.; 253: 2010:3191-3198
3. Griffith DE, Aksamit T, Brown-Elliott BA, Catanzaro A, Daley C, Gordin F, Holland SM, Horsburgh R, Huitt G, Iademarco MF, et al. An official ATS/ IDSA statement: diagnosis, treatment, and prevention of nontuberculous mycobacterial diseases. Am J Respir Crit Care Med. 2007;175(4):367-416. 
4. Holland SM. Nontuberculous mycobacteria.AmJMed Sci. 2001;321:4955. doi:10.1097/00000441-20010100000008.

5. Murcia M, León C, de la Hoz F, Saravia J. Asociación Micobacterias-VIH/ SIDA en Pacientes Atendidos en un Hospital Universitario en Bogotá, Colombia. Revista de Salud Pública. 2007;9(1):97-105. doi:10.1590/ s0124-00642007000100010.

6. McFarland EJ, Kuritzkes DR. Clinical features and treatment of infection due to mycobacterium fortuitum/chelonae complex. Curr Clin Top Infect Dis. 1993;13:188-202.

7. Verweij KE, Kamerik AR, van Ingen J, et al. Application of modern microbiological diagnostic methods for tuberculosis in Macha, Zambia. Int J Tuberc Lung Dis. 2010;14:1127-31.

8. Desikan P, Tiwari K, Panwalkar N, et al. Public health relevance of nontuberculous mycobacteria among AFB positive sputa. Germs. 2017;7(1):1018. doi:10.18683/germs.2017.1103. Disponible en:
9. Farnia P, Ghanavi J, Tabasri P, Saif $S$, Velayati A. The importance of single nucleotide polymorphisms in interferon gamma receptor-1 gene in pulmonary patients infected with rapid grower mycobacterium. 2017. Disponible en: http://www. sciencedirect.com/science/article/pii/ S2212553116301777

10. Porat MD, Austin MS. Bilateral knee periprosthetic infection with Mycobacterium fortuitum. J Arthroplasty. 2008;23:787-9.

11. Madhusudhan N, Malini A, Sangma MMB. A Case of Surgical Site Infection Caused by Mycobacterium fortuitum, following Herniorrhaphy. Journal of Clinical and Diagnostic Research: JCDR. 2016;10(11):DD01-DD02. doi:10.7860/ JCDR/2016/20045.8924.

12. Brown-Elliott BA, Vasireddy $\mathrm{S}$, Vasireddy $\mathrm{R}$, et al. Utility of Sequencing the erm(41) Gene in Isolates of Mycobacterium abscessus subsp. abscessus with Low and Intermediate Clarithromycin MICs. Journal of Clinical Microbiology.
2015;53(4):1211-1215. doi:10.1128/ JCM.02950-14.

13. Brown-Elliott BA, Nash KA, Wallace RJ. Antimicrobial Susceptibility Testing, Drug Resistance Mechanisms, and Therapy of Infections with Nontuberculous Mycobacteria. Clinical Microbiology Reviews. 2012;25(3):545582. doi:10.1128/CMR.05030-11. Disponible en:

14. Abbass K, Adnan MK, Markert RJ, Emig M, Khan NA. Mycobacterium fortuitum breast abscess after nipple piercing. Canadian Family Physician. 2014;60(1):51-52.

Correspondencia: Manuel Fernando Sanchez Herrera

Direccion: Calle El Incario 238 dpto 201 El Derby-Santiago de Surco

Telefono: 980200004

Correo electronico:manuel.sanchez@upch.pe

Suscríbete a nuestro canal de Youlillhe y disfruta la mejor y más completa información de investigación científica

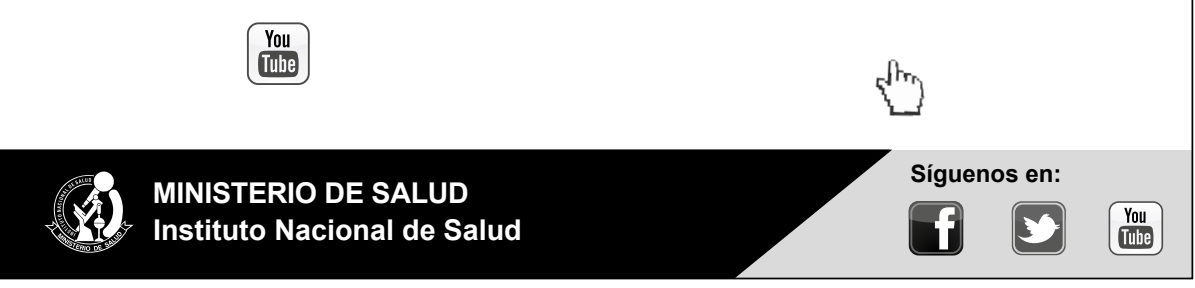

